\title{
PARITY VIOLATION AND ELECTRIC DIPOLE MOMENTS IN ATOMS AND MOLECULES
}

\author{
V. A. DZUBA \\ School of Physics, University of New South Wales \\ Sydney 2052, Australia \\ V.Dzuba@unsw.edu.au \\ V. V. FLAMBAUM \\ School of Physics, University of New South Wales \\ Sydney 2052, Australia \\ V.Flambaum@unsw.edu.au \\ Received Day Month Year \\ Revised Day Month Year
}

\begin{abstract}
We review the current status of the study of parity and time invariance violation in atoms, nuclei and molecules. We focus on parity non-conservation in cesium and three of the most promising areas of research: (i) parity non-conservation in a chain of isotopes, (ii) search for nuclear anapole moments, and (iii) search for permanent electric dipole moments (EDMs) of atoms and molecules, which in turn are caused by either an electron EDM or nuclear T, $P$-odd moments such as a nuclear EDM or nuclear Schiff moment.

Keywords: Parity violation; Electric dipole moment; EDM; Schiff moment.
\end{abstract}

PACS numbers:11.30.Er; 12.15.Ji; 31.15.A-

\section{Introduction}

The study of parity and time invariance violation at low-energy in atoms, molecules and nuclei is a relatively inexpensive alternative to high-energy searches for new physics beyond the standard model (see, e.g. reviews 1, 2). Accurate measurements of the parity non-conservation (PNC) in atoms is one of the most promising ways of exploring this path. It culminated in a very precise measurement of the PNC in the cesium atom. ${ }^{3}$ There was even an indication that this measurement showed some disagreement with the standard model and might indeed lead to new physics. ${ }^{4}$ It took considerable efforts of several groups of theorists to improve the interpretation of the measurement and resolve the disagreement in favor of the standard model. The disagreement was removed when the Breit ${ }^{5}$ and quantum electrodynamic corrections $\sqrt{6}$ were included and the accuracy of the treatment of atomic correlations was improved! $7 \sqrt{8}$

The latter work ${ }^{8}$ claimed a very small theoretical uncertainty of just $0.26 \%$. Combined with the PNC measurement ${ }^{3}$ it leads to perfect agreement between the 
measured weak nuclear charge of the ${ }^{133} \mathrm{Cs}$ nucleus and the value predicted by the standard model. It also puts strong constraints on new physics beyond it.

The PNC amplitude calculated in Ref. 8 is about $0.9 \%$ smaller than that of earlier calculations. ${ }^{7}[11$ The difference is significant given that all earlier works agree very well with each other and theoretical uncertainty in them is estimated to be $0.4-0.5 \%$ 남 7 The effect of this difference on the agreement with the standard model is not large, however, there are important implications for the constraints on new physics beyond it. The difference was attributed in Ref. 8 to the role of high-order correlations. However, recently in Ref. 12 it was found that the authors of Ref. 8 overlooked some important correlation contributions to the PNC amplitude. The uncertainty of $0.26 \%$ claimed in Ref. 8 is based mostly on analysis of the dominating contributions. The uncertainty due to the correlation corrections in other terms has been underestimated. The result of Ref. 8 when corrected as suggested in Ref. 12 is in perfect agreement with the previous calculations. ${ }^{7}[9]$ $\mathrm{PNC}$ in Cs is discussed in more detail in the next section.

It is unlikely that any new measurements of the PNC in atoms can compete with the cesium experiment in accuracy of the interpretation. There are conflicting tendencies in the accuracy of the measurements and the calculations. On the one hand, it is easier to get highly accurate measurements for heavy atomic systems where the PNC effect is larger. On the other hand, advanced computational techniques favor light alkaline atoms. The best accuracy for both calculations and measurements have been achieved for cesium atom: $0.35 \%$ for the measurements ${ }^{3}$ and $0.4-0.5 \%$ for the calculations. ${ }^{[7][12]} \mathrm{A}$ higher accuracy of the interpretation is possible for the rubidium atom, which is a lighter analog of cesium. The rubidium atom has been considered so far for a different type of PNC effect: the nuclear spindependent PNC in the ground-state hyperfine transition, which is mostly due to the nuclear anapole moment ${ }^{133}$ (see section 4). For the spin-dependent PNC accurate calculations are also possible, but less important than for measurements of the weak nuclear charge. It is unclear whether the weak nuclear charge of rubidium can be measured to high accuracy. A heavier analog of cesium, the francium atom, is considered for both types of PNC measurements, the effect of weak nuclear charge and the anapole moment 13 The accuracy of calculations for francium could be, at best, as good as for cesium, but may be slightly lower due to the larger relativistic effects which increase the Breit, quantum electrodynamics (QED) and neutron distribution ("neutron skin") corrections. There are suggestions to measure PNC in $s-d$ transitions of $\mathrm{Ba}^{+15[16}$ and $\mathrm{Ra}^{+16] 17}$ ions which have an electronic structure similar to that of the cesium atom. Here again the accuracy of the calculations is unlikely to outperform cesium due to larger correlations in $d$-states (only $s$ and $p$ states are involved in cesium). Note that some improvement here may be achieved using experimental values of the electromagnetic E1 $p-d$ amplitudes in the sum over intermediate $p$ states in the calculation of the $s-d$ PNC amplitude ${ }^{16}$.

However, there are areas of symmetry breaking in atomic systems where we do 
Table 1. Correlated PNC amplitude in Cs [in $10^{-11} i\left(-Q_{W} / N\right)$ a.u.] in different calculations. Breit, QED and neutron skin corrections are not included.

\begin{tabular}{ll}
\hline \multicolumn{1}{c}{ Value } & \multicolumn{1}{c}{ Source and method } \\
\hline $0.908(9)$ & CP+PTSCI, Ref. 9] \\
$0.909(9)$ & CC SD, Ref. [10] \\
$0.905(9)$ & MBPT with fitting, Ref. 11] \\
$0.9078(45)$ & CP+PTSCI, Ref. 7] \\
$0.8998(24)$ & CC SDvT, Ref. [8 \\
\hline
\end{tabular}

not need very high accuracy of atomic calculations: (i) the PNC measurements for a chain of isotopes; (ii) the measurements of nuclear anapole moments; and (iii) the measurements of the P,T-odd permanent electric dipole moments of atoms and molecules. Accurate numerical atomic calculations are not needed for the interpretation of the PNC measurements for a chain of isotopes since the change of the atomic wave function is very small and may be estimated analytically in the ratio of PNC effects for different isotopes. The interpretations of the anapole and electric dipole moment measurements do rely on atomic or molecular calculations. However, high accuracy here is not as important as for the measurements of the weak nuclear charge (where we search for a small deviation from the Standard model predictions).

Below we briefly review parity non-conservation in cesium, and each of the three subjects listed above.

\section{Parity non-conservation in cesium}

Parity non-conservation in cesium is currently the best low-energy test of the electroweak theory. It is due to the high accuracy of the measurements and its interpretation. The experimental value for the PNC amplitude is ${ }^{3}$

$$
\operatorname{Im}\left(E_{\mathrm{PNC}}\right) / \beta=1.5935(56) \mathrm{mV} / \mathrm{cm} \text {. }
$$

where $\beta$ is the vector transition amplitude. The amplitude $E_{\mathrm{PNC}}$ is proportional to the weak nuclear charge $Q_{W}$

$$
E_{\mathrm{PNC}}=K\left(-Q_{W} / N\right),
$$

where $K$ is the electron structure factor which comes from atomic calculations, $N$ is the number of neutrons $\left(N=78\right.$ for $\left.{ }^{133} \mathrm{Cs}\right)$. The main challenge in the calculation of the PNC amplitude is an accurate treatment of the inter-electron correlations. There are also minor contributions arising due to the effects of the Breit interaction, quantum electrodynamics and neutron skin corrections. We first consider the dominant term (the correlated PNC amplitude) and add minor corrections later.

Table 1 shows the most accurate values of the correlated amplitude found in different calculations. The abbreviation $\mathrm{CP}+\mathrm{PTSCI}$ stands for the correlation potential ${ }^{18}$ combined with the perturbation theory in screened Coulomb interaction, 
Table 2. All significant contributions to the $E_{\mathrm{PNC}}$ [in $10^{-11} i\left(-Q_{W} / N\right)$ a.u.] for Cs.

\begin{tabular}{|c|c|c|c|}
\hline \multirow[t]{2}{*}{ Contribution } & \multicolumn{2}{|c|}{ Value } & \multirow{2}{*}{ Source } \\
\hline & Ref. 8 & Ref. 12 & \\
\hline$\overline{\text { Core }(n<6)}$ & $-0.0020(2)$ & $0.0018(8)$ & Ref. 8,12 \\
\hline Main $(n=6-9)$ & \multicolumn{2}{|c|}{$0.8823(17)$} & Ref. 8 \\
\hline Tail $(n>9)$ & $0.0195(20)$ & $0.0238(35)$ & Ref. 8.12 \\
\hline Subtotal & $0.8998(25)$ & $0.9079(40)$ & Ref. 8,12 \\
\hline Breit & \multicolumn{2}{|c|}{$-0.0055(1)$} & Ref. 5 \\
\hline QED & \multicolumn{2}{|c|}{$-0.0029(3)$} & Ref. 6] \\
\hline Neutron skin & \multicolumn{2}{|c|}{$-0.0018(5)$} & Ref. 5 \\
\hline Total & $0.8906(26)$ & $0.8977(40)$ & Ref. 8,12 \\
\hline
\end{tabular}

CC SD stands for the coupled cluster with single and double excitations, CC SDvT means the coupled cluster with single, double and valence triple excitations. One can see that all results except those of Ref. 8 agree with each other within $0.4 \%$. The result of Ref. 8 is $0.9 \%$ smaller than another most accurate result of Ref. 7. Table 2 shows the reason for this difference. The authors of Ref. 8 used the sum-over-states approach in which the PNC amplitude is expressed as a sum over complete set of single-electron states, including states in the core and states above the core. Contribution of each state is calculated independently of others and the use of the same approximation for all terms is practically impossible. A very sophisticated coupled cluster method was used to calculate the main term (contributions of the four lowest states above the core). Significantly less accurate approximation was used for the tail (contribution of the states above $9 p$ ) and a very simple Hartree-Fock approximation was used for the core. The uncertainty of just $10 \%$ was assigned for the sum of the core and tail contributions. However, as it was shown in Ref. 12 the inclusion of the core polarization effect for the core contribution changes even the sign of the core contribution brining the total change to about $200 \%$, far beyond the declared $10 \%$ uncertainty. The tail contribution is also significantly larger when the Brueckner-type correlations are included. When the core and the tail contributions are corrected as suggested in Ref. 12, the result of Ref. 8 comes into excellent agreement with the previous calculations (see "Subtotal" line in Table 2 and Table 1. the corresponding numbers are 0.9079 and 0.9078 ).

Table 2 also lists all other significant contributions to the PNC amplitude in cesium. The resulting PNC amplitude is

$$
E_{\mathrm{PNC}}=0.8977(45) \times 10^{-11} i\left(-Q_{W} / N\right) .
$$

Note that the uncertainty coming from the analysis of Ref. 12 is smaller $( \pm 0.004$, see Table 2) due to the small uncertainty of the main term claimed in Ref. 8 , For a conservative estimate we use in (3) a slightly larger uncertainty of Ref. 7. The central points of Ref. 7 and Ref. 12 are practically identical.

To find the value of the weak nuclear charge of ${ }^{133} \mathrm{Cs}$ from (1) one also needs the value for the vector transition amplitude $\beta$. The most accurate value for $\beta$ comes 
from the analysis $\frac{19}{19}$ of the Bennett and Wieman measurements 20

$$
\beta=26.957(51) a_{B}^{3},
$$

where $a_{B}$ is the Bohr radius. Comparing (3), (11) and (4) leads to

$$
Q_{W}\left({ }^{133} \mathrm{Cs}\right)=-72.58(29)_{\text {expt }}(36)_{\text {theory }} .
$$

This value is in a reasonable agreement with the prediction of the standard model, $Q_{W}^{\mathrm{SM}}=-73.23(2)^{21}$ (see also Refs. 22, 23, 24). If we add theoretical and experimental errors in (5) in quadrature, the Cs PNC result deviates from the standard model value by $1.4 \sigma$ :

$$
Q_{W}-Q_{M}^{\mathrm{SM}} \equiv \delta Q_{W}=0.65(46) .
$$

For small deviations from the Standard Model values we may relate this to the deviation in $\sin ^{2} \theta_{W}$ using the simple relationship $\delta Q_{W} \approx-4 Z \delta\left(\sin ^{2} \theta_{W}\right)$ which gives $\delta\left(\sin ^{2} \theta_{W}\right)=-0.0030(21)$ and

$$
\sin ^{2} \theta_{W}=0.2356(21) .
$$

This is $1.4 \sigma$ off the standard model value $0.2386(1)^{21}$ at near zero momentum transfer.

The new physics originated through vacuum polarization of the gauge boson propagators, and is described by the weak isospin conserving $S$ and isospin breaking $T$ parameters 23

$$
Q_{W}-Q_{M}^{\mathrm{SM}}=-0.800 S-0.007 T .
$$

At the $1 \sigma$ level (6) leads to $S=-0.81(58)$.

Finally, a positive $\Delta Q_{W}$ could also be indicative of an extra $Z$ boson in the weak interaction 24

$$
Q_{W}-Q_{M}^{\mathrm{SM}} \approx 0.4(2 N+Z)\left(M_{W} / M_{Z \chi}\right)^{2} .
$$

Using (6) leads to $M_{Z_{\chi}}>700 \mathrm{GeV} / \mathrm{c}^{2}$.

\section{Chain of isotopes}

It is convenient in this section to present the values measured in atomic PNCexperiments in a form similar to (2)

$$
E_{P N C}=k_{P N C} Q_{W},
$$

where $k_{P N C}$ is an electron structure factor which comes from atomic calculations, and $Q_{W}$ is the weak nuclear charge. Very sophisticated calculations are needed for accurate interpretation of the measurements as has been discussed in the introduction and previous section. An alternative approach was suggested in Ref. 25. If the same PNC effect is measured for at least two different isotopes of the same atom than the ratio

$$
\mathcal{R}=\frac{E_{P N C}^{\prime}}{E_{P N C}}=\frac{Q_{W}^{\prime}}{Q_{W}}
$$


of the PNC signals for the two isotopes does not depend on the electron structure factor. It was pointed out however in Ref. 26 that possible constraints on the new physics coming from the isotope ratio measurements are sensitive to the uncertainties in the neutron distribution which are sufficiently large to be a strong limitation factor on the value of such measurements. The problem was addressed in Ref. 27 and more recently in Ref. 28. The authors of Ref. 27 argue that experimental data on neutron distribution, such as, e.g. the data from the experiments with antiprotonic atoms, ${ }^{29}$ can be used to reduce the uncertainty. In the more general approach of Ref. 28 nuclear calculations are used to demonstrate that the neutron distributions are correlated for different isotopes. This leads to significant cancelations of the relevant uncertainties in the ratio (11).

The parameter $\mathcal{F}$ of the sensitivity of the ratio (11) to new physics can be presented in the form

$$
\mathcal{F}=\frac{h_{p}}{h_{0}}=\left(\frac{\mathcal{R}}{\mathcal{R}_{0}}-1\right) \frac{N N^{\prime}}{Z \Delta N}
$$

where $h_{p}$ is the new physics coupling to protons $\left(\Delta Q_{\text {new }}=Z h_{p}+N h_{n}\right), h_{0}$ comes from the $\mathrm{SM}, \mathcal{R}_{0}$ is the ratio (11) assuming that each isotope has the same proton and neutron distribution (no neutron skin), $N$ and $N^{\prime}$ are the numbers of neutrons in two isotopes, $Z$ is the number of protons and $\Delta N=N^{\prime}-N$. The constraints on new physics parameter $h_{p}$ are affected by the experimental error $\delta \mathcal{R}_{\exp }$ and uncertainties in $\mathcal{R}_{0}$ due to insufficient knowledge of neutron distributions. However, as is argued in Ref. 28, these errors are correlated and strongly cancel each other. Indeed, the distribution of the core neutrons is nearly the same in different isotopes. Significant changes happen in a small number of valence nucleons only. Estimations of Ref. 28 show that corresponding contribution to $\delta \mathcal{F}$ is in the range $10^{-3} \div 10^{-2}$ which is about an order of magnitude smaller than the uncorrelated one. In the end, the isotope-chain measurements may be more sensitive to new physics than current parity-violating electron scattering measurements ${ }^{30}$ (by a factor of 10 for such atoms as Cs, Ba and Dy).

Experiments on isotope chains are in progress at Berkeley for Dy and $\mathrm{Yb}$ atoms, $\stackrel{31,32}{32}$ at TRIUMF for Fr atoms, $\frac{14}{,}$ at Los Alamos for $\mathrm{Yb}^{+}$ions, $\frac{33}{33}$ and at Groningen (KVI) for $\mathrm{Ra}^{+}$ions ${ }^{17}$ There is an interesting recent suggestion to mea-

sure the $\mathrm{PNC}$ in metastable $\mathrm{Xe}$ and $\mathrm{Hg} \cdot{ }^{34}$ Most of these experiments consider also the measurements of the nuclear anapole moment.

\section{Anapole moment}

The notion of the anapole moment was introduced by B. Ya. Zeldovich! ${ }^{35}$ Nuclear anapole moment (AM) is the magnetic $P$ and $C$-odd, $T$-even nuclear moment caused by the $P$-odd weak nuclear forces. Interaction of electrons with AM magnetic field (which may be called the PNC hyperfine interaction) dominates the nuclear-spindependent contribution to the atomic or molecular PNC effect. 
First calculations of the nuclear AM and proposals for experimental measurements were presented in Refs. 36. 40. Corrections to the AM interaction with electrons due to finite nuclear size were considered in Ref. 41, The status of the nuclear AM many-body calculations is presented in reviews 1, 2, The authors of Refs. 38, 39 (see also Ref. 42) note in particular that the nuclear-spin-dependent PNC effect is strongly enhanced in diatomic molecules due to mixing of the close rotational states of opposite parity including mixing of $\Lambda$ or $\Omega$ doublets. The PNC effects produced by the weak charge are not enhanced. Therefore the AM effect dominates PNC in molecules. This greatly simplifies the detection of AM in diatomic molecules compared to atoms. In atoms the AM effect is 50 times smaller than the weak charge effect; AM effect is separated as a small difference of the PNC effects in different hyperfine transitions. A review of the parity and time invariance violation in diatomic molecules (including the AM effect) can be found in Ref. 43 .

The idea of the AM contribution enhancement may be explained as follows. After the averaging over electron wave function the effective operator acting on the angular variables may contain three vectors: the direction of molecular axis $\mathbf{N}$, the electron angular momentum $\mathbf{J}$ and nuclear spin I. Scalar products NI and NJ are $T$-odd and $P$-odd. Therefore, they are produced by the $T, P$-odd interactions discussed in the next section. $P$-odd, $T$-even operator $V_{P}$ must be proportional to $\mathbf{N}[\mathbf{J} \times \mathbf{I}]$. It contains nuclear spin $\mathbf{I}$, therefore, the weak charge does not contribute. The nuclear AM is directed along the nuclear spin $\mathbf{I}$, therefore, it contributes to $V_{P}$. The matrix elements of $\mathbf{N}$ between molecular rotational states are well-known, they produce rotational electric dipole transitions in polar molecules. Therefore, $V_{P}$ (induced by the magnetic interaction of the nuclear AM with molecular electrons) mixes close rotational-hyperfine states of opposite parity. The interval between these levels is five orders of magnitude smaller than the interval between the opposite parity levels in atoms (by the factor $m_{e} / M$ where $m_{e}$ and $M$ are the electron and reduced molecular masses), therefore PNC effects are five orders of magnitude larger. Further enhancement may be achieved by a reduction of the intervals by an external magnetic field. $\underline{39}$

The effect is further enhanced for heavy molecules. It grows with the nuclear charge as $Z^{2} A^{2 / 3} R(Z \alpha)$, where $R(Z \alpha)$ is the relativistic factor which grows from $R=1$ at low $Z$ to $R \sim 10$ for $Z>80$ and the factor $A^{2 / 3}$ comes from the nuclear anapole, $A$ is the nucleon number. Good candidates for the measurements include the molecules and molecular ions with the projection of the electron angular momentum on the molecular axis $\Omega=\Lambda_{z}+\Sigma_{z}=1 / 2$, i.e. in $\Sigma_{1 / 2}\left(\Lambda_{z}=0\right)$ or $\Pi_{1 / 2}$ $\left(\Lambda_{z}=1\right)$ electronic ground states $\frac{38}{39}$ for example, $\mathrm{YbF}, \mathrm{BaF}, \mathrm{HgF}, \mathrm{PbF}, \mathrm{LaO}$, $\mathrm{LuO}, \mathrm{LaS}, \mathrm{LuS}, \mathrm{BiO}, \mathrm{BiS}, \mathrm{YbO}+, \mathrm{PbO}+, \mathrm{BaO}+, \mathrm{HgO}+$, etc. Molecular experiments are currently in progress at Yale ${ }^{44}$ and Groningen KVI $\stackrel{45}{4}$ An interesting idea of studying AM contribution to the NMR spectra of chiral molecules was discussed in Ref. 46.

Interpretation of the AM measurements requires electron structure calculations. A number of semiempirical and $a b$ initio calculations have been performed for di- 
atomic molecules of experimental interests in Refs. 47.57, So far the only nuclear $\mathrm{AM}$ which has been measured is the $\mathrm{AM}$ of the ${ }^{133} \mathrm{Cs}$ nucleus. It is done by comparing PNC amplitudes between different hyperfine structure sublevels in the same PNC experiment where the Cs weak charge is measured ${ }^{3}$ (the method was proposed in $\frac{36}{6}$ ). Interpretation of the measurements 58 indicates some problems. For example, the value of Cs AM is inconsistent with the limit on the AM of Tl.

To resolve the inconsistencies and obtain valuable information about P-odd nuclear forces it would be very important to measure anapole moments for other nuclei. In particular, it is important to measure AM for a nucleus with an unpaired neutron (Cs and $\mathrm{Tl}$ have unpaired protons). AM of such nucleus depend on different combination of the weak interaction constants providing important cross-check. Good candidates for such measurements include odd isotopes of Ra, Dy, Pb, Ba, La, $\mathrm{Lu}$ and $\mathrm{Yb}$. The Ra atom has an extra advantage because of a strong enhancement of the spin-dependent PNC effect in the ${ }^{1} \mathrm{~S}_{0}-{ }^{3} \mathrm{D}_{2}$ transition due to proximity of the opposite-parity state ${ }^{3} \mathrm{P}_{1}^{o}\left(\Delta E=5 \mathrm{~cm}^{-1}\right), 60$

Experimental work is in progress for Rb and Fr at TRIUMF 13 14 and for Dy and $\mathrm{Yb}$ at Berkeley ${ }^{31 \mid 32]}$ Measurements for Xe and $\mathrm{Hg}$ are planned at university of Crete $\stackrel{34}{34}$

Calculations of the nuclear spin-dependent PNC amplitudes (including the effect of $\mathrm{AM}$ ) for a number of atoms and ions of experimental interest were reported in Refs 60.70 .

\section{Electric dipole moment}

Permanent electric dipole moment (EDM) of neutron, atom or molecule would violate both $P$ and $T$ invariance. Under conditions of the $C P T$-theorem this would also mean $C P$-violation. The Kobayashi-Maskawa mechanism of the standard model leads to extremely small values of the EDMs of the particles. It is also too weak to explain the matter-antimatter asymmetry of the Universe. On the other hand, most of the popular extensions to the standard model predict much larger EDMs which are within experimental reach. The EDM of an atom or a molecule is mostly due to either electron EDM and T,P-odd electron-nucleon interactions in paramagnetic systems (with non-zero total electron angular momentum $J$ ) or to the $T, P$-odd nuclear forces in diamagnetic systems $(J=0$; nuclear-spin-dependent e-N interaction contributes here too). The existence of the $T, P$-odd nuclear forces leads to the $T, P$-odd nuclear moments in the expansion of the nuclear potential in powers of the distance $R$ from the center of the nucleus. The lowest-order term in the expansion, the nuclear EDM, is unobservable in neutral atoms due to the total screening of the external electric field by atomic electrons. It might be possible however to observe the nuclear EDM in ions (see below). The first non-vanishing electrostatic terms which survives the screening in neutral systems are the octupole moment and the Schiff moment. The octupole moment does not produce EDM in diamagnetic atoms. Below we discuss the effects of the nuclear and electron EDM and the Schiff 
moment.

\subsection{Nuclear EDM}

It was widely believed that one needs neutral particles (e.g., neutron, neutral atom or molecule) to study EDMs. This is because the EDM is expected to be very small and it would be very hard to see the effect of its interaction with external electric field on the background of the much stronger interaction with the electric charge. On the other hand, the EDM of neutral systems is very much suppressed by the effect of screening of an external electric field by electrons (Schiff theorem - see section 5.3). The Schiff theorem may be violated by the relativistic effect (which dominates in the case of the electron EDM), the hyperfine interaction and the nuclear finite size effect ${ }^{71}$ For example, the lowest-order $T, P$-odd nuclear moment, the nuclear EDM is practically unobservable in the neutral systems (except for a small contribution due to the hyperfine interaction calculated in Ref. 72). First observable $T, P$-odd nuclear moment, the Schiff moment, is non-zero due to the finite nuclear size.

It is important therefore to explore the possibility of studying EDMs of charged particles (e.g. muons or atomic ions). There are realistic suggestions of this kind in Refs. 73 76 based on the use of ion storage rings. There are also proposals to measure EDM in molecular ions. ${ }^{77}$

The external electric field is not totally screened on the nucleus of an ion. Its value is

$$
E_{N}=\frac{Z_{i}}{Z} E_{0},
$$

where $E_{0}$ is the external electric field, $E_{N}$ is the electric field at the nucleus, $Z e$ is the nuclear charge, $Z_{i} e$ is the charge of the ion, $e$ is proton charge. The formula (13) can be obtained in a very simple way. The second Newton law for the ion and its nucleus in the electric filed reads

$$
M_{i} a_{i}=Z_{i} e E_{0}, M_{N} a_{N}=Z e E_{N},
$$

where $M_{i}$ is the ion's mass, $a_{i}$ is its acceleration, $M_{N}$ is nuclear mass $\left(M_{N} \approx\right.$ $\left.M_{i}\right)$, and $a_{N}$ is its acceleration. Since the ion and its nucleus move together, the accelerations must be the same $\left(a_{i}=a_{N}\right)$, therefore

$$
E_{N}=\frac{Z_{i}}{Z} E_{0} \frac{M_{N}}{M_{i}} \approx \frac{Z_{i}}{Z} E_{0} .
$$

Quantum mechanical derivations of this formula can be found in Refs. 78, 80 , Numerical calculations of the screened electric field inside an atomic ion were performed in a number of our works (see, e.g. Ref. 78).

The Hamiltonian of the nuclear EDM $\left(d_{N}\right)$ interaction with the electric field is given by

$$
\hat{H}_{d}=d_{N} E_{N}=d_{N} \frac{Z_{i}}{Z} E_{0} .
$$


Screening is stronger for diatomic molecules where we have an additional suppression factor in eq. (14), $M_{N} / M_{i}=M_{1} /\left(M_{1}+M_{2}\right)$, where $M_{1}$ and $M_{2}$ are the masses of the first and second nucleus. For the average electric field acting on the first nucleus we obtain

$$
E_{1 N}=\frac{Z_{i}}{Z_{1}} \frac{M_{1}}{M_{1}+M_{2}} E_{0}
$$

A Quantum mechanical derivation of this formula can be found in Ref. 80, Note that the screening factor here contains both nuclear masses. This indicates that the nuclear motion can not be ignored and the screening problem is more complicated than in atoms. For example, in a naive ionic model of a neutral polar molecule $A^{+} B^{-}$, both ions $A^{+}$and $B^{-}$should be located in the area of zero (totally screened) electric field since their average acceleration is zero. This could make $A^{+}$and $B^{-}$ EDM unobservable even if they are produced by the nuclear Schiff moment or electron EDM. In a more realistic molecular calculations the Schiff moment and electron EDM effects are not zero, however, they may be significantly suppressed (in comparison with a naive estimate of ionic EDM in a very strong field of another ion) and the results of the calculations may be unstable.

Note also that contrary to atomic ions the contribution of the Schiff moment to the T,P-odd effects in heavy molecular ions exceeds the contribution of the nuclear EDM 80

In the case of monochromatic external electric field its frequency can be chosen to be in resonance with the atomic electron excitation energy. Then for the effective Hamiltonian we can have

$$
\hat{H}_{d}=d_{N} E_{N}(t) \gg d_{N} E_{0} .
$$

\subsection{Electron EDM}

Paramagnetic atoms and molecules which have an unpaired electron are most sensitive to the electron EDM. The EDM of such systems can be expressed in the form

$$
d=K d_{e},
$$

where $d$ is the EDM of an atom or molecule, $d_{e}$ is electron EDM, and $K$ is electron structure factor which comes from atomic calculations. The factor $K$ increases with nuclear charge $Z$ as $Z^{3[81}$ times large relativistic factor $R(Z \alpha)^{82}$ which may exceed the value of 3 in heavy atoms. A rough estimate of the enhancement factor in heavy atoms with external $s_{1 / 2}$ or $p_{1 / 2}$ electron is $K \sim 3 Z^{3} \alpha^{2} R(Z \alpha) \sim 10^{2}-10^{3}$.

Several orders of magnitude larger $K \sim 10^{7}-10^{11}$ exist in molecules due to the mixing of the close rotational levels of opposite parity (including $\Lambda$-doublets). .38 Following Sandars this enhancement factor is usually presented as a ratio of a very large internal molecular field to the external electric field which polarizes the molecule. 
The best current limit on electron EDM comes from the measurements of the thallium $\mathrm{EDM}^{83}$ and reads

$$
d_{e}=(6.9 \pm 7.4) \times 10^{-28} e \mathrm{~cm} .
$$

Here the value $K=-585^{\sqrt{84}}$ was used for the interpretation of the measurements. The value of $K$ for $\mathrm{Tl}$ is very sensitive to the inter-electron correlations but three most complete calculations 84 give very close results (there are also calculations of Ref. 87 which give smaller result).

In contrast to paramagnetic atoms the diamagnetic (closed shell) atoms are much less sensitive to electron EDM. This is because the only possible direction of the atomic EDM in this case is along nuclear spin and hyperfine structure interaction must be involved to link electron EDM to nuclear spin. For example, for the mercury atom $K \sim 10^{-2}[88 \mid 89]$ However, due to very strong constraints on the mercury $\mathrm{EDM}^{90}$ the limit on electron EDM extracted from these measurements is competitive to the $\mathrm{Tl}$ result $(19)^{90}$

$$
\left|d_{e}\right|<3 \times 10^{-27} e \mathrm{~cm} \text {. }
$$

New strong limits on the electron EDM were recently found from the measurements for the $\mathrm{YbF}$ molecule. ${ }^{91}$ Experiments are in progress to measure the electron $\mathrm{EDM}$ in $\mathrm{Cs},{ }^{92} \mathrm{Fr},{ }^{93} \mathrm{ThO},{ }^{94} \mathrm{PbO}^{95}$ and in solid-state experiments. ${ }^{96}$

Molecular EDM due to electron EDM were calculated for a number of diatomic molecules in Refs $97,[105$.

\subsection{Schiff moment}

Schiff moment is the lowest-order $T, P$-odd nuclear moment which appears in the expansion of the nuclear potential when screening of the electric field by atomic electrons is taken into account. This potential can be written as (see different derivations, e.g. in Ref. 106, 107, 80, 2)

$$
\phi(\mathbf{R})=\int \frac{e \rho(r)}{|\mathbf{R}-\mathbf{r}|} d^{3} r+\frac{1}{Z}(\mathbf{d} \cdot \nabla) \int \frac{\rho(r)}{|\mathbf{R}-\mathbf{r}|} d^{3} r,
$$

where $\rho(\mathbf{r})$ is the nuclear charge density normalized to $Z$, and $\mathbf{d}$ is the nuclear EDM. The second term in (21) is the screening. Taking into account the finite nuclear size the dipole term in the multipole expansion of (21) can be written as ${ }^{108}$

$$
\psi(\mathbf{R})=-\frac{3 \mathbf{S} \cdot \mathbf{R}}{B} \rho(R),
$$

where $B=\int \rho(R) R^{4} d r$ and

$$
\mathbf{S}=\frac{e}{10}\left[\left\langle r^{2} \mathbf{r}\right\rangle-\frac{5}{3 Z}\left\langle r^{2}\right\rangle\langle\mathbf{r}\rangle\right]
$$

is the Schiff moment. The expression (22) has no singularities and can be used in relativistic calculations. More accurate expressions, which include corrections for 
Table 3. EDMs of diamagnetic atoms of experimental interest.

\begin{tabular}{|c|c|c|c|c|}
\hline$Z$ & Atom & $\begin{array}{l}{\left[S /\left(e \mathrm{fm}^{3}\right)\right]} \\
\times 10^{-17} e \mathrm{~cm}\end{array}$ & $\begin{array}{l}\eta e \mathrm{~cm} \\
\times 10^{-25}\end{array}$ & Experiment \\
\hline 2 & ${ }^{3} \mathrm{He}$ & $8 \times 10^{-5}$ & $5 \times 10^{-4}$ & \\
\hline 54 & ${ }^{129} \mathrm{Xe}$ & 0.38 & 0.7 & $\begin{array}{l}\text { Seattle } \frac{119}{11} \text { Ann Arbol } 120 \\
\text { Princetor } 121\end{array}$ \\
\hline 70 & ${ }^{171} \mathrm{Yb}$ & -1.9 & 3 & Bangalore ${ }^{122}$ Kyotd 123 \\
\hline 80 & ${ }^{199} \mathrm{Hg}$ & -2.8 & 4 & Seattle 90 \\
\hline 86 & ${ }^{223} \mathrm{Rn}$ & 3.3 & 3300 & TRIUMF 124 \\
\hline 88 & ${ }^{225} \mathrm{Ra}$ & -8.2 & 2500 & Argonne 125 KV】126 \\
\hline 88 & ${ }^{223} \mathrm{Ra}$ & -8.2 & 3400 & \\
\hline
\end{tabular}

the finite nuclear size $\sim Z^{2} \alpha^{2}$, were obtained in Refs. 108, 80, 109. The authors also considered a partial screening of the octupole moment 109 The Schiff moment is caused by the $T, P$-odd nuclear forces. The dominant mechanism is believed to be the $T, P$-odd nucleon-nucleon interaction. Another important contribution comes from the EDMs of protons and neutrons.

Schiff moment is the dominant nuclear contribution to the EDM of diamagnetic atoms and molecules. The best limit on the EDM of diamagnetic atoms comes from the measurements of the EDM of mercury performed in Seattle ${ }^{90}$

$$
\left|d\left({ }^{199} \mathrm{Hg}\right)\right|<3.1 \times 10^{-29}|e| \mathrm{cm} .
$$

Interpretation of the measurements requires atomic and nuclear calculations. Atomic calculations link the EDM of the atom to its nuclear Schiff moment. Nuclear calculations relate Schiff moment to the parameters of the $T, P$-odd nuclear interactions. Summary of atomic ${ }^{110}\left[112\right.$ and nuclear ${ }^{113][14 \mid 116}$ calculations for diamagnetic atoms of experimental interest is presented in Table 3 . To compare the EDM of different atoms we present only the results of our nuclear calculations which all were performed by the same method. For $\mathrm{Hg}$ and $\mathrm{Ra}$ there are several recent nuclear many-body calculations available (see references in the most recent calculation 117 ).

The dimensionless constant $\eta$ characterizes the strength of the $P, T$-odd nucleonnucleon interaction (in units of the weak interaction Fermi constant $G$ ) which is to be determined from the EDM measurements. Using (24) and the data from the Table one can get

$$
S\left({ }^{199} \mathrm{Hg}\right)=(-1.8 \pm 4.6 \pm 2.7) \times 10^{-13} e \mathrm{~cm}
$$

and for the $T, P$-odd neutron-proton interaction

$$
\eta_{n p}=(1 \pm 3 \pm 2) \times 10^{-5} .
$$

\subsection{Nuclear enhancement.}

In some nuclei with the quadrupole deformation there is a close opposite parity level with the same angular momentum. This leads to an enhancement of nuclear 
EDM ${ }^{115}$ and Schiff moment $\sqrt{113}$ up to an order of magnitude. Significantly larger enhancement is possible in nuclei with the octupole deformation. 116 This can be explained in a very simple way. Nuclear deformation creates a collective intrinsic Schiff moment in the rotating nuclear reference frame

$$
S_{\text {intr }} \approx e Z R_{N}^{3} \frac{9 \beta_{2} \beta_{3}}{20 \pi \sqrt{35}}
$$

where $R_{N}$ is the nuclear radius, $\beta_{2} \approx 0.2$ is the parameter of the quadrupole deformation, and $\beta_{3} \approx 0.1$ is the parameter of the octupole deformation. The intrinsic Schiff moment (27) does not violate $T$ or $P$ invariance and, if no $T, P$ odd interaction is present, it averages to zero in the laboratory reference frame due to the nuclear rotation. However, when $T, P$-odd interaction is included, it can mix close rotational states of opposite parity (which are similar to $\Omega$ - doublet in diatomic molecules). Small energy interval between these states leads to a strong enhancement of the nuclear Schiff moment in the laboratory reference frame

$$
S_{l a b} \sim \frac{\left\langle+\left|H_{P T}\right|-\right\rangle}{E_{+}-E_{-}} S_{i n t r} \sim 0.05 e \beta_{2} \beta_{3}^{2} Z A^{2 / 3} \eta r_{0}^{3} \frac{\mathrm{eV}}{E_{+}-E_{-}} \sim 700 \times 10^{-8} \eta e \mathrm{fm}^{3},
$$

where $r_{0}=1.2 \mathrm{fm}$ is the inter-nucleon distance $\left|E_{+}-E_{-}\right| \sim 50 \mathrm{keV}$. The estimate (28) is about 500 times larger than the Schiff moment of a spherical nucleus like $\mathrm{Hg}$.

It was pointed out in Ref. 118 that the octupole deformation doesn't need to be static. Soft octupole vibrations lead to similar enhancement.

Large values of the Schiff moment for Ra and Rn (see Table 3) are due to the nuclear octupole deformation. Some additional enhancement of the atomic EDM is due to the large nuclear charge. The nuclear enhancement may also manifest itself in molecules, e.g. in $\mathrm{RaO}, \frac{127}{12}$ where the $T, P$-odd nuclear spin- molecular axis interaction exceeds that in the experimentally studied $\mathrm{TlF}^{110}[128$ about 500 times.

\section{Acknowledgments}

This work was supported by the Australian Research Council.

\section{References}

1. J. S. M. Ginges and V. V. Flambaum, Phys. Rep. 397 (2004) 63.

2. V. F. Dmitriev and I.B. Khriplovich, Phys. Rep. 391 (2004) 243. bibitemDF V.F. Dmitriev and I.B. Khriplovich, Phys. Rep. 391 (2004) 243. Updated in arxiv: nucl-th/030983v3.

3. C. S. Wood, S. C. Bennett, D. Cho, B. P. Masterson, J. L. Roberst, C. E. Tanner, C. E. Wieman, Science 275 (1997) 1759.

4. S. C. Bennett and C. E. Wieman, Phys. Rev. Lett. 82 (1999) 2484; Phys. Rev. Lett. 82 (1999) 4153(E); Phys. Rev. Lett. 83 (1999) 889(E).

5. A. Derevianko, Phys. Rev. Lett. 85 (2000) 1618; Phys. Rev. A 65 (2001) 012106. V. A. Dzuba, C. Harabati, W. R. Johnson, and M. S. Safronova, Phys. Rev. A 63 (20001); 
M. G. Kozlov, S. G. Porsev, and I. I. Tupitsyn, Phys. Rev. Lett. 86 (2001) 3260; V. A. Dzuba, V. V. Flambaum, and M. S. Safronova, Phys. Rev. A 73 (2006) 022112.

6. A. I. Milstein and O.P. Sushkov, Phys. Rev. A 66 (2002) 022108; W. R. Johnson, I. Bednyakov, and G. Soff, Phys. Rev. Lett. 87 (2001) 233001; Phys. Rev. Lett. 88 (2002) 079903(E); M. Yu. Kuchiev and V. V. Flambaum, Phys. Rev. Lett. 89 (2002) 283002; A. I. Milstein, O. P. Sushkov, and I. S. Terekhov, Phys. Rev. Lett. 89 (2002) 283003; M. Yu. Kuchiev, J. Phys. B 35 (2002) 4101; A. I. Milstein, O. P. Sushkov, and I. S. Terekhov, Phys. Rev. A 67 (2003) 062103; J. Sapirstein, K. Pachucki, A. Veitia, and K. T. Cheng, Phys. Rev. A 67 (2003) 052110; M. Yu. Kuchiev and V. V. Flambaum, J. Phys. B 36 (2003) R191; V. M. Shabaev, K. Pachucki, I. I. Tupitsyn, and V. A. Yerokhin, Phys. Rev. Lett. 94 (2005) 213002 (2005); V. V. Flambaum and J. S. M Ginges, Phys. Rev. A 72 (2005) 052115.

7. V. A. Dzuba, V. V. Flambaum, and J. S. M. Ginges, Phys. Rev. D 66 (2002) 076013.

8. S. G. Porsev, K. Beloy, and A. Derevianko, Phys. Rev. Lett. 102 (2009) 181601.

9. V. A. Dzuba, V. V. Flambaum, O. P. Sushkov, Phys. Lett. A 141 (1989) 147.

10. S. A. Blundell, W. R. Johnson, and J. Sapirstein, Phys. Rev. Lett. 65 (1990) 1411; S. A. Blundell, J. Sapirstein, and W. R. Johnson, Phys. Rev. D 45 (1992) 1602.

11. M. G. Kozlov, S. G. Porsev, and I. I. Tupitsyn, Phys. Rev. Lett. 86 (2001) 3260.

12. V. A. Dzuba, J. C. Berengut, V. V. Flambaum, B. Roberts, arXiv:1207.5864.

13. D. Sheng, L. A. Orozco, and E. Gomez, J. Phys. B 43 (2010) 074004.

14. E. Gomez, L. A. Orozco, and G. D. Sprouse, Rep. Prog. Phys. 69 (2006) 79; D. Sheng, J. Zhang, L. A. Orozco, Rev. Scient. Instr. 83 (2012) 043106.

15. N. Fortson, Phys. Rev. Lett. 70 (1993) 2383.

16. V. A. Dzuba, V. V. Flambaum, J. S. M. Ginges, Phys. Rev. A 63 (2001) 062101.

17. L. W. Wansbeek, B. K. Sahoo, R. G. E. Timmermans, K. Jungmann, B. P. Das, and D. Mukherjee, Phys. Rev. A 78 (2008) 050501.

18. V. A. Dzuba, V. V. Flambaum, P. G. Silvestrov, O. P. Sushkov, J. Phys. B 20 (1987) 1399.

19. V. A. Dzuba and V. V. Flambaum, Phys. Rev. A 62 (2000) 052101.

20. S. C. Bennett and C. E. Wieman, Phys. Rev. Lett. 82 (1999) 2484.

21. J. Beringer et al (Particle Data Group), Phys. Rev. D 86 (2012) 010001.

22. P. G. Blunden, W. Melnitchouk and A. W. Thomas, arxiv: 1208.4310.

23. J. L. Rosner, Phys. Rev. D 65 (2002) 073026.

24. W. J. Marciano and J. L. Rosner, Phys. Rev. Lett. 65 (1990) 2963; 68 (1992) 898(E).

25. V. A. Dzuba, V. V. Flambaum, and I.B. Khriplovich, Z. Phys. D 1 (1986) 243.

26. E. N. Fortson, Y. Pang, and L. Wilets, Phys. Rev. Lett. 65 (1990) 2857.

27. A. Derevianko and S. G. Porsev, Phys. Rev. A 65 (2002) 052115.

28. B. A. Brown, A. Derevianko, and V. V. Flambaum, Phys. Rev. C 79 (2009) 035501.

29. A. Trzcinska, J. Jastrzebski, P. Lubinski, F.J. Hartmann, R. Schmidt, T. von Egidy, and B. Klos, Phys. Rev. Lett. 87 (2001) 082501.

30. R. D. Young, R. D. Carlini, A. W. Thomas, and J. Roche, Phys. Rev. Lett. 99 (2007) 122003.

31. A. T. Nguyen, D. Budker D, D. DeMille, and M. Zolotorev, Phys. Rev. A 56 (1997) 3453.

32. K. Tsigutkin, D. Dounas-Frazer, A. Family, J. E. Stalnaker, V. V. Yashchuk, and D. Budker, Phys. Rev. Lett. 103 (2009) 071601.

33. J. Torgerson, private communication (2010).

34. L. Bougas, G. E. Katsoprinakis, W. von Klitzing, J. Sapirstein, and T. P. Rakitzis, Phys. Rev. Lett. 108 (2012) 210801.

35. Ya. B. Zeldovich, Zh. Eksp. Teor. Fiz. 33 (1957) [Sov. Phys. JETP 6 (1958) 1184]. 
36. V. V. Flambaum and I.B. Khriplovich. ZhETF 79 (1980) 1656 [Sov. Phys. JETP 52 (1980) 835].

37. V. V. Flambaum, I. B. Khriplovich, and O. P. Sushkov, Phys. Lett. B. 146 (1984) 367.

38. O. P. Sushkov and V. V. Flambaum, ZhETF 75 (1978) 1208 [Sov. Phys. JETP 48 (1978) 608].

39. V. V. Flambaum and I. B. Khriplovich, Phys. Lett. A 110 (1985) 121.

40. W.C. Haxton, E.M. Henley, M.J. Musolf, Phys. Rev. Lett. 63 (1989) 949.

41. V. V. Flambaum and C. Hanhart, Phys. Rev. C 48 (1993) 1329.

42. L. N. Labzovsky, Zh. Eksp. Teor. Fiz. 75 (1978) 856 [Sov. Phys. JETP 48 (1978) 434].

43. M. G. Kozlov and L. N. Labzowsky, J. Phys. B 28 (1995) 1933.

44. D. DeMille, S. B. Cahn, D. Murphree, D. A. Rahmlow, and M. G. Kozlov, Phys. Rev. Lett. 100 (2008) 023003.

45. T. A. Isaev, S. Hoekstra, and R. Berger, Phys. Rev. A 82 (2010) 052521.

46. S Nahrwold and R. Berger, J. Phys. Chem. 130 (2009) 214101; G. Laubender and R. Berger, ChemPhysChem 4 (2003) 395.

47. D. DeMille, S. B. Cahn, D. Murphree, D. A. Rahmlow, and M. G. Kozlov, Phys. Rev. Lett. 100 (2008) 023003.

48. M. K. Nayak and B. P. Das, Phys. Rev. A 79 (2009) 060502.

49. G. Kozlov, A. V. Titov, N. S. Mosyagin, and P. V. Souchko, Phys. Rev. A 56 (1997) R3326.

50. M. G. Kozlov and L. N. Labzowsky, J. Phys. B 28 (1995) 1933.

51. A. V. Titov, N. S. Mosyagin, and V. F. Ezhov, Phys. Rev. Lett. 77 (1996) 5346.

52. Y. Y. Dmitriev, Y. G. Khait, M. G. Kozlov, L. N. Labzovsky, A. O. Mitrushenkov, A. V. Shtoff, and A. V. Titov, Phys. Lett. A 167 (1992) 280.

53. M. Kozlov, V. Fomichev, Y. Dmitriev, L. Labzovsky, and A. Titov, J. Phys. B 20 (1987) 4939.

54. K. I. Baklanov, A. N. Petrov, A. V. Titov, and M. G. Kozlov, Phys. Rev. A 82 (2010) 060501(R).

55. T. A. Isaev, S. Hoekstra, and R. Berger, Phys. Rev. A 82 (2010) 052521.

56. A. Borschevsky, M. Ilias, V. A. Dzuba, K. Beloy, V. V. Flambaum, and P. Schwerdtfeger, Phys. Rev. A 85 (2012) 052509.

57. T. A. Isaev, R. Berger, arxiv: 1206.6013.

58. V. V. Flambaum and D. W. Murray, Phys. Rev. C 56 (1997) 1641.

59. P. A. Vetter, D. M. Meekhof, P. K. Majumder, S. K. Lamoreaux, and E. N. Fortson, Phys. Rev. Lett. 74 (1995) 2658.

60. V. V. Flambaum, Phys. Rev. A 60 (1999) R2661; V. A. Dzuba, V. V. Flambaum, and J. S. M. Ginges, Phys. Rev. A 61 (2000) 062509.

61. S. G. Porsev and M. G. Kozlov, Phys. Rev. A 64 (2001) 064101.

62. W. R. Johnson, M. S. Safronova, and U. I. Safronova, Phys. Rev. A 67 (2003) 062106.

63. V. A. Dzuba and V. V. Flambaum, Phys. Rev. A 81 (2010) 052515.

64. A. D. Singh and B. P. Das, J. Phys. B 32 (1999) 4905.

65. S. G. Porsev, M. G. Kozlov, and Yu. G. Rakhlina, Hyperfine Interactions 127 (2000) 395.

66. V. A. Dzuba and V. V. Flambaum, Phys. Rev. A 83 (2011) 042514.

67. V. A. Dzuba and V. V. Flambaum, Phys. Rev. A 83 (2011) 052513.

68. S. G. Porsev, M. S. Safronova, and M. G. Kozlov Phys. Rev. A 86 (2012) 022504.

69. V. A. Dzuba, V. V. Flambaum, and C. Harabati, Phys. Rev. A 84 (2011) 052108.

70. V. A. Dzuba and V. V. Flambaum, Phys. Rev. A 85 (2012) 012515. 
71. I.I. Schiff, Phys. Rev. 132 (1963) 2194.

72. S.G. Porsev, J.S.M. Ginges, V.V. Flambaum Phys. Rev. A 83 (2011) 042507.

73. I.B. Khriplovich, Phys. Lett. B 444 (1998) 98; Nucl. Phys. A 663-664 (2000) 147.

74. F. J. M. Farley et al, Phys. Rev. Lett. 93 (2004) 052001.

75. Y. F. Orlov, W. M. Morse, and Y. K. Semertzidis, Phys. Rev. Lett. 96 (2006) 214802.

76. V. G. Baryshevsky, J. Phys. G 35 (2008) 035102.

77. A. E. Leanhardt, J. L. Bohn, H. Loh, P. Maletinsky, E. R. Meyer, L. C. Sinclair, R.P. Stutz, E. A. Cornell, arxiv: 1008.2997v3.

78. V. A. Dzuba, V. V. Flambaum, P. G. Silvestrov, O. P. Sushkov, Phys. Lett. A 131 (1988) 461.

79. S. Oshima, Phys. Rev. C 81 (2010) 038501.

80. V. V. Flambaum and A. Kozlov, Phys. Rev. A 85 (2012) 022505.

81. P. G. H. Sandars, Phys. Lett. 14 (1965) 194; P. G. H. Sandars, Phys. Lett. 22 (1966) 290.

82. V. V. Flambaum, Yad. Fiz. 24 (1976) 383 [Sov. J. Nucl. Phys. 24 (1976) 199].

83. B. C. Regan, E. D. Commins, C. J. Schmidt, and D. DeMille, Phys. Rev. Lett. 88 (2002) 071805.

84. Z. W. Liu and H. P. Kelly, Phys. Rev. A 45 (1992) R4210.

85. V. A. Dzuba and V. V. Flambaum, Phys. Rev. A 80 (2009) 062509.

86. S. G. Porsev, M. S. Safronova, and M. G. Kozlov Phys. Rev. Lett. 108 (2012) 173001.

87. H. S. Nataraj, B. K. Sahoo, B. P. Das, and D. Mukherjee, Phys. Rev. Lett. 106 (2011) 200403.

88. V. V. Flambaum and I. B. Khriplovich, Zh. Eksp. Teor. Fiz. 89 (1985) 1505 [Sov. Phys. JETP 62 (1985) 872].

89. A.-M. Mårtensson-Pendrill and P. Öster, Phys. Scripta 36 (1987) 444.

90. W. C. Griffith, M. D. Swallows, T. H. Loftus, M. V. Romalis, B. R. Heckel, and E. N. Fortson, Phys. Rev. Lett. 102 (2009) 101601.

91. J. J. Hudson, D. M. Kara, I. J. Smallman, B. E. Sauer, M. R. Tarbutt, and E. A. Hinds, Nature 473 (2011) 493.

92. J. M. Amini, C. T. Munger, and H. Gould, Phys. Rev. A 75 (2007) 063416; H. Gould, Int. J. Mod. Phys. D 16 (2007) 2337.

93. H. Kawamura et al, unpublished; Y. Sakemi, unpublished; S. Sanguinetti et al, Opt. Lett. 34 (2009) 893.

94. A. C. Vutha et al, J. Phys. B 43 (2010) 074007.

95. A. V. Titov, N. S. Mosyagin, A. N. Petrov, T. A. Isaev, and D. P. DeMille, Progr. Theor. Chem. Phys. 15 (2006) 253.

96. B. J. Heidenreich et al, Phys. Rev. Lett. 95 (2005) 253004; D. Budker, S. K. Lamoreaux, A. O. Sushkov, and O. P. Sushkov, Phys. Rev. A 73 (2006) 022107; A. O. Sushkov, S. Eckel, and S. K. Lamoreaux, Phys. Rev. A 79 (2009) 022118.

97. E. R. Meyer and J. L. Bohn, Phys. Rev. A 78 (2008) 010502(R).

98. M. K. Nayak and R. K. Chaudhuri, Phys. Rev. A 78 (2008) 012506.

99. H. M. Quiney, H. Skaane, and I. P. Grant, J. Phys. B 31 (1998) L85.

100. M. G. Kozlov and V. F. Ezhov, Phys. Rev. A 49 (1994) 4502.

101. A. V. Titov, N. S. Mosyagin, and V. F. Ezhov, Phys. Rev. Lett. 77 (1996) 5346.

102. M. G. Kozlov, J. Phys. B 30 (1997) L607.

103. F. A. Parpia, J. Phys. B 31 (1998) 1409.

104. N. Mosyagin, M. G. Kozlov, and A. Titov, J. Phys. B 31 (1998) L763.

105. A. N. Petrov, A. V. Titov, T. A. Isaev, N. S. Mosyagin, and D. DeMille, Phys. Rev. A 72 (2005) 022505.

106. V. Spevak, N. Auerbach, and V. V. Flambaum, Phys. Rev. C 56 (1997) 1357. 
107. R. A. Sen'kov, N. Auerbach, V. V. Flambaum, and V. G. Zelevinsky, Phys. Rev. A 77 (2008) 014101.

108. V. V. Flambaum and J. S. M. Ginges, Phys. Rev. A 65 (2002) 032113.

109. V. V. Flambaum and A. Kozlov, Phys. Rev. C 85 (2012) 068502.

110. V. A. Dzuba, V. V. Flambaum, J. S. M. Ginges, and M. G. Kozlov, Phys. Rev. A 66 (2002) 012111.

111. V. A. Dzuba, V. V. Flambaum, and J. S. M. Ginges, Phys. Rev. A 76 (2007) 034501.

112. V. A. Dzuba, V. V. Flambaum, and S. G. Porsev, Phys. Rev. A 80 (2009) 032120.

113. O. P. Sushkov, V. V. Flambaum, and I. B. Khriplovich, ZhETF 87 (1984) 1521 [JETP 60 (1984) 873].

114. V. V. Flambaum, I. B. Khripovich, and O. P. Sushkov, Nucl. Phys. A 449 (1986) 750 .

115. W.C. Haxton, and E.M. Henley Phys. Rev. Lett. 51 (1983) 1937.

116. N. Auerbach, V. V. Flambaum, and V. Spevak, Phys. Rev. Lett. 76 (1996) 4316; V. Spevak, N. Auerbach, and V. V. Flambaum, Phys. Rev. C 56 (1997) 1357.

117. S. Ban, J. Dobaczewski, J. Engel, A. Shukla, Phys. Rev. C 82 (2010) 015501.

118. J. Engel, J. L. Friar, and A. C. Hayes, Phys. Rev. C 61 (2000) 035502; V. V. Flambaum and V. G. Zelevinsky, Phys. Rev. C 68 (2003) 035502; N. Auerbach, V. F. Dmitriev, V. V. Flambaum, A. Lisetskiy A, R. A. Sen'kov, and V. G. Zelevinsky, Phys. Rev. C 74 (2006) 025502; N. Auerbach, V. F. Dmitriev, V. V. Flambaum, A. Lisetskiy A, R. A. Sen'kov, and V. G. Zelevinsky, Phys. At. Nuc. 70 (2007) 1654.

119. T. G. Vold, F. J. Raab, B. Heckel, and E. N. Fortson, Phys. Rev. Lett. 52 (1984) 2229.

120. M. A. Rosenberry and T. E. Chupp, Phys. Rev. Lett. 86 (2001) 22.

121. M. V. Romalis and M. P. Ledbetter, Phys. Rev. Lett. 87 (2001) 067601.

122. V. Natarajan, Eur. Phys. J. D 32 (2005) 33.

123. T. Takano, M. Fuyama, H. Yamamoto, and Y. Takahashi (unpublished).

124. E. R. Tardiff et al, Nucl. Instr. Meth. Phys. Res. D 579 (2007) 472.

125. http://www.phy.anl.gov/mep/atta/research/radiumedm.html

126. H. W. Wilschut et al, Pramana J. Phys. 75 (2010) 163.

127. V. V. Flambaum, Phys. Rev. A 77 (2008) 024501.

128. D. Cho, K. Sangster, and E. A. Hinds, Phys. Rev. A 44 (1991) 2783. 